\title{
Expression of CD180 in common B cell neoplasms using immunohistochemistry
}

\author{
Ahmad T. Mansour ${ }^{1,2}$ (D) $\cdot$ Tamadur A. Mahasneh $^{3} \cdot$ Sahar Mustafa $^{1} \cdot$ Sarah Shawash $^{3} \cdot$ Omar Jaber $^{3} \cdot$ Tariq N. Adily $^{1}$ \\ Received: 18 May 2020 / Accepted: 5 October 2020 / Published online: 13 October 2020 \\ (C) Springer-Verlag GmbH Germany, part of Springer Nature 2020
}

\begin{abstract}
Diagnosis of low-grade B cell lymphoma can be challenging especially on limited cytological and histological samples, CD180 is Toll-like receptor that was found to be of value in the diagnosis of marginal zone lymphoma using flow cytometry. In this pilot study, we examine the expression of CD180, using an immunohistochemical method, on a number of common B cell lymphoproliferative neoplasms. Immunohistochemical staining for sixty-seven cases of five B cell neoplasms, specifically, marginal zone lymphoma, mantle cell lymphoma, diffuse large B cell lymphoma, follicular lymphoma, and chronic lymphocytic leukemia/small lymphocytic lymphoma was performed on archived, paraffin-embedded, formalin-fixed tissue. CD180 expression is preserved in all cases of marginal zone lymphoma, sixteen out of 17 cases of follicular lymphoma cases, and none of the diffuse large B cell lymphoma or chronic lymphocytic leukemia/small lymphocytic lymphoma cases. One in 12 cases of mantle cell lymphoma shows weak and focal staining. Using CD180 immunohistochemical stain can be valuable and offer some help in the diagnosis of B cell neoplasms, especially on small tissue samples.
\end{abstract}

Keywords Marginal zone lymphoma $\cdot$ CD180 $\cdot$ Low-grade B cell lymphoma $\cdot$ Immunohistochemistry

\section{Introduction}

CD180 is a member of the Toll-like receptor (TLR) homolog that belongs to the TLR4 family. This protein was thought to be present normally on B lymphocytes only; however, subsequent studies have demonstrated this antigen on monocytes and myeloid dendritic cells but not plasmacytoid dendritic cells [1]. Interestingly, B cells, which harbor the highest concentration of this protein, are devoid of other TLR4 proteins [2]. Targeting CD180 with anti-CD180 results in rapid proliferation and differentiation of $\mathrm{B}$ cells, increased production of various antibodies, affinity maturation, and immunological memories [3, 4].

Ahmad T. Mansour

mansouau@ucmail.uc.edu

1 Department of Pathology, Microbiology, and Forensic Medicine-School of Medicine, University of Jordan, Queen Rania Street, Amman 11942, Jordan

2 Department of Pathology and Laboratory Medicine, University of Cincinnati College of Medicine, 234 Goodman Street, OH 45219 Cincinnati, USA

3 Department of Pathology, King Hussein Cancer Center, Queen Rania Street, Amman 11942, Jordan
Previous studies have described an underexpression of this protein in several B cell lymphoproliferative neoplasms, with the exception of marginal zone lymphoma. Additionally, CD180 is lost on the circulating cells of follicular lymphoma but preserved on follicular lymphoma cells prepared from lymph node tissue [5-8]. Those studies were performed using flow cytometry to characterize CD180 expression on different $\mathrm{B}$ cell neoplasms. In this pilot study, we try to elucidate the expression of CD180 on several common mature B cell neoplasms using immunohistochemistry hoping it would help subclassify B cell tumors using formalin-fixed paraffin-embedded tissue. This, in turn, would expand the use of this marker to the general pathology laboratories where flow cytometry setup or expertise might not be available. Furthermore, this study demonstrates the feasibility of using this marker on small tissue samples as well as archived material, obviating the need for fresh samples.

\section{Methods}

\section{Patient samples}

Sixty-seven cases of common B cell lymphomas were retrieved from the archived materials in Jordan University 
Hospital and King Hussein Cancer Center. Diagnoses were reviewed and confirmed by two hematopathologists (AM and TA) using the most recent update on the World Health Organization Classification of Tumors of Hematopoietic and Lymphoid tissue. Formalin-fixed, paraffin-embedded blocks were retrieved and $4-\mu \mathrm{m}$ unstained slides were cut on each case. The study was approved by Internal Review Boards at the Jordan University Hospital and King Hussein Cancer Center.

\section{Immunohistochemistry}

Four-micron-thick tissue sections were cut from paraffinembedded tissue blocks and placed on clean, charged glass slides. Paraffin sections were dried by placing in an oven at $70{ }^{\circ} \mathrm{C}$ for $20 \mathrm{~min}$. The slides were dewaxed in two changes of xylene for $5 \mathrm{~min}$ each, rehydrated through a series of graded concentrations of alcohol, and washed twice with distilled water for $5 \mathrm{~min}$ to remove residual alcohol. The antigen retrieval process was performed by placing the slide container in EDTA buffer solution, $\mathrm{pH} 8$, for $15 \mathrm{~min}$ at $95{ }^{\circ} \mathrm{C}$ using microwave heating. After heating, the slides were cooled at room temperature for $30 \mathrm{~min}$ and were briefly washed with phosphate-buffered saline (PBS; Ph 7.3 \pm 0.1 ) for $5 \mathrm{~min}$. The slides were treated with $3 \%$ hydrogen peroxide in distilled water for $5 \mathrm{~min}$ to block endogenous peroxidase activity. After that, slides were washed in PBS and treated with Power Block ${ }^{\mathrm{TM}}$ reagent for $10 \mathrm{~min}$. The slides were then incubated with the primary monoclonal CD180 antibody (1:250 dilution; RP105/CD180, NBP1-76705, Novus Biologica, CO, USA) for $1.5 \mathrm{~h}$ at room temperature. Following washing in PBS, secondary antibody (BioGenex) was applied for $30 \mathrm{~min}$. After incubation, slides were rinsed in PBS and then incubated with Polymer-HRP Reagent for $30 \mathrm{~min}$ at room temperature and rinsed in PBS. Subsequently, the slides were incubated with the diaminobenzidine chromogen reagent for $10 \mathrm{~min}$, and then washed in distilled water. Hematoxylin was applied as a counterstain for $3 \mathrm{~min}$ and then washed with tap water. Finally, the slides were dehydrated in a series of ascending concentrations of alcohol and mounted with DPX reagent. Negative controls for CD180 monoclonal antibody were performed without applying the primary antibody while the positive control represents tonsillar tissue sections (Fig. 1).

\section{Results}

A total of sixty-seven cases were studied as follows: thirteen cases of marginal zone lymphoma (MZL) ( 9 splenic MZL and 4 extranodal MZL), 17 cases of follicular lymphoma (all cases were grade 1 with centrocyte count ranging from 2 to $5 / 10$ high power fields), 12 cases of mantle cell lymphoma, 13 cases of diffuse large B cell lymphoma, and 12 cases of chronic lymphocytic leukemia/small lymphocytic lymphoma (10 bone marrow and 2 lymph nodes). All MZL cases stained positively for CD180 (more than $90 \%$ of the cells with strong, circumferential membranous staining in 11 cases) (Fig. 2); notably, 2 extranodal MZL cases showed focal positivity ( $60 \%$ of cells). Sixteen out of 17 FL cases were also positive (> $90 \%$ of the cells with strong, circumferential membranous staining in 11 cases and partial staining in the remaining 5) (Fig. 3). Only 1 in 12 MCL cases showed focal weak staining, estimated at $\sim 10 \%$; the remainder of the cases were completely negative (Fig. 4). In contrast, none of the CLL/SLL (Fig. 5) or DLBCL cases were positive. The pattern of staining is membranous and circumferential with cytoplasmic perinuclear accentuation; this pattern was noted in the positive specimens as well as the tissue controls. Table 1 is a summary of the findings.

\section{Discussion}

Marginal zone lymphoma (MZL) is divided, according to the 2017 update of the World Health Organization (WHO) classification of tumors of hematopoietic and lymphoid tissues into three major categories, splenic MZL, nodal MZL, and extranodal MZL of mucosa-associated lymphoid tissue [9]. A pediatric MZL is added as a provisional entity in the latest edition of the WHO book as a subset of nodal marginal zone lymphoma that has distinctive clinical and morphological features. The diagnosis of marginal zone lymphoma is usually a diagnosis of exclusion, as there is no specific marker for rendering this diagnosis with satisfying certainty, and it is best entertained after ruling out other subtypes of low-grade B cell lymphoma. While the immunoglobulin superfamily receptor translocation-associated 1 (IRTA1) showed some promise in this regard, it was found to be positive in other tumor types, and to be negative in a significant number of marginal zone lymphoma of different types, limiting its use in routine practice [10].

In their pioneering studies, Miguet et al. and MayeurRousse et al. [5, 6] have shown, using flow cytometry, that CD180 is underexpressed on the plasma membranes of neoplastic cells in various B cell tumors, with the notable exception of marginal zone lymphoma. The tumors studied in these published works included chronic lymphocytic leukemia (CLL), mantle cell lymphoma (MCL), lymphoplasmacytic lymphoma (LPL), follicular lymphoma (FL), hairy cell leukemia (HCL), and marginal zone lymphoma (MZL). In 2018, a study by Wu et al. [7] demonstrated that splenic diffuse red pulp lymphoma (SDRPL) cells also showed preserved expression of CD180, a similar finding was found previously in 8 patients with SDPRL by Miguet et al. [5]. Interestingly, cellular preparation of follicular lymphoma derived from lymph node, not circulating neoplastic cells, shows preserved 
Fig. 1 Control. a Tonsil with follicular hyperplasia

(hematoxylin and eosin), b CD180 highlighting B cells in the mantle zone, notice the faint staining of B cells in the germinal center (original magnification $\times$ 400)
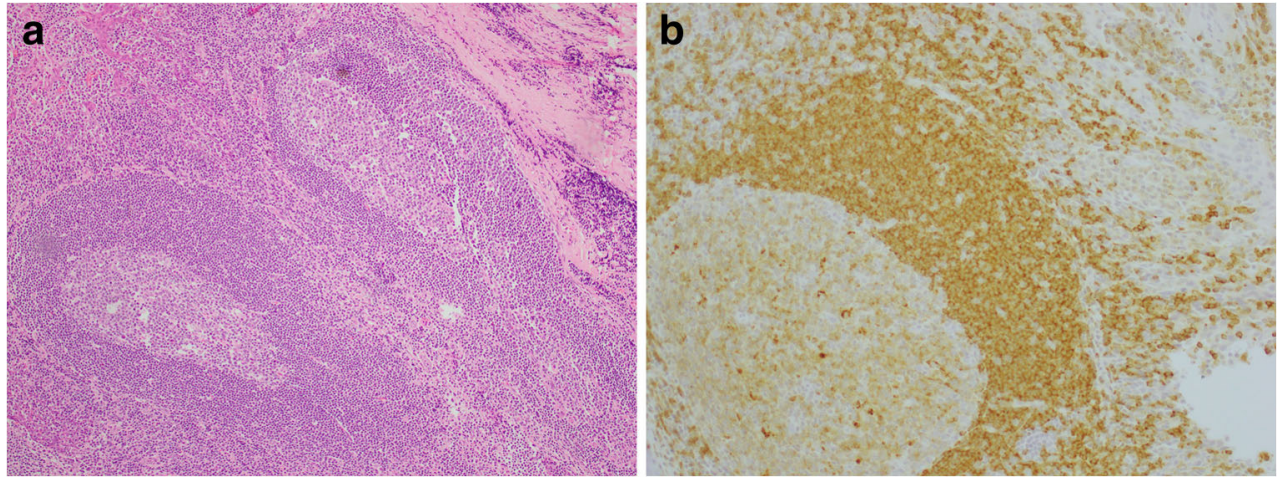

expression of CD180 [8]. All the previous studies were performed using flow cytometry; to the best of our knowledge, this is the first time an immunohistochemical assay on formalin-fixed, paraffin-embedded tissue is performed.

Our study has shown that CD180 is consistently positive in all marginal zone lymphoma cases studied. Additionally, follicular lymphoma shows preserved CD180 expression in 16 of 17 cases (94\%); five cases of FL (29\%), however, show weak or partial expression, estimated at $\sim 50 \%$ of the cells. All cases of diffuse large B cell lymphoma as well as chronic lymphocytic leukemia/small lymphocytic lymphoma are negative for CD180. The majority of mantle cell lymphoma cases (11/12) are completely negative. Our findings lend credence to the results published by other authors who used flow cytometry.

Furthermore, our study has expanded the scope of CD180 expression on one other type of MZL, namely, extranodal marginal zone lymphoma of mucosa-associated lymphoid tissue (MALT lymphoma).

The demand for rendering the diagnosis of lymphoma on small tissue samples is increasing. With the accelerating development and availability of molecular techniques to aid in the diagnosis, the expectation is to give more with less; the treating clinicians now expect an expanding plethora of morphologic, immunophenotypic, prognostic, and therapeutic information based on an ever-decreasing sample size.
The diagnosis of lymphoma relies on cytological, histological, immunophenotypic, and genetic/molecular features [9]. The use of small samples, including needle core biopsies and aspirations, is increasing. Compared to excisional biopsies, these less invasive techniques are more tolerated by the patients, cause less pain, and result in fewer postoperative complications, especially in deep-seated lesions. One study has shown a cost saving of approximately $75 \%$ compared to excisional biopsies [11]. Interestingly, low diagnostic accuracy was noted with marginal zone lymphoma using needle core biopsy samples compared to other types of B cell lymphoma [12]. We believe that the judicious use of CD180 immunohistochemical stain will reliably increase the diagnostic accuracy of MZL.

Diagnosis and subclassification of low-grade B cell lymphoma on small tissue cores can be challenging. While some types of lymphoma have immunohistochemical markers with excellent specifities for diagnosis, such as cyclin D1 and SOX11 for mantle cell lymphoma and LEF1 for chronic lymphocytic leukemia, marginal zone lymphoma lacks such a specific immunohistochemical marker.

Given the heterogeneity of morphology characterizing members of the marginal zone lymphoma family on one hand,

Table 1 Summary of CD180 expression in common B cell lymphoma

\begin{tabular}{|c|c|c|c|}
\hline Histologic type & No. of positive cases (\%) & $\begin{array}{l}\text { Percentage of } \\
\text { positive cells }\end{array}$ & Pattern of staining \\
\hline Marginal zone lymphoma & $13 / 13(100 \%)$ & $\begin{array}{l}12 \text { cases }>90 \% \\
1 \text { case } \sim 60 \%\end{array}$ & $\begin{array}{l}\text { All cases show strong membranous and cytoplasmic positivity } \\
\text { with perinuclear accentuation }\end{array}$ \\
\hline Follicular lymphoma & $16 / 17(94 \%)$ & $\begin{array}{l}11 \text { cases }>90 \% \\
5 \text { cases } \sim 50 \%\end{array}$ & $\begin{array}{l}11(65 \%) \text { cases show strong membranous and cytoplasmic } \\
\text { positivity with perinuclear accentuation; } 5 \text { (29\%) cases } \\
\text { show weak and partial staining }\end{array}$ \\
\hline Mantel cell lymphoma & $0 / 12(0 \%)$ & $\begin{array}{l}1 \text { case } \sim 10 \% \\
11 \text { cases, } 0 \%\end{array}$ & One case shows weak and incomplete membranous staining \\
\hline $\begin{array}{l}\text { Chronic lymphocytic lymphoma/small } \\
\text { lymphocytic lymphoma }\end{array}$ & $0 / 12(0 \%)$ & $0 \%$ & No discernable staining was noted \\
\hline Diffuse large B cell lymphoma & $0 / 13(0 \%)$ & $0 \%$ & No discernable staining was noted \\
\hline
\end{tabular}


Fig. 2 Marginal zone lymphoma. a A section from a splenic marginal zone lymphoma (hematoxylin and eosin), b expression of CD180 is strong and diffuse, $\mathbf{c}$ the cells are negative for CD5 (note the background $\mathrm{T}$ cells), $\mathbf{d}$ the cells are negative for $\mathrm{CD} 10$. (original magnification $\times 400$ )
Fig. 3 Follicular lymphoma. a Follicular lymphoma

(hematoxylin and eosin), note the two neoplastic follicles composed mostly of centrocytes, $\mathbf{b}$ the cells are positive for CD20, $\mathbf{c}$ the cells are positive for $\mathrm{CD} 10$, and $\mathbf{d}$ BCL2. e Expression of CD180 in the neoplastic cells in follicular lymphoma (original magnification $\times 400$ )
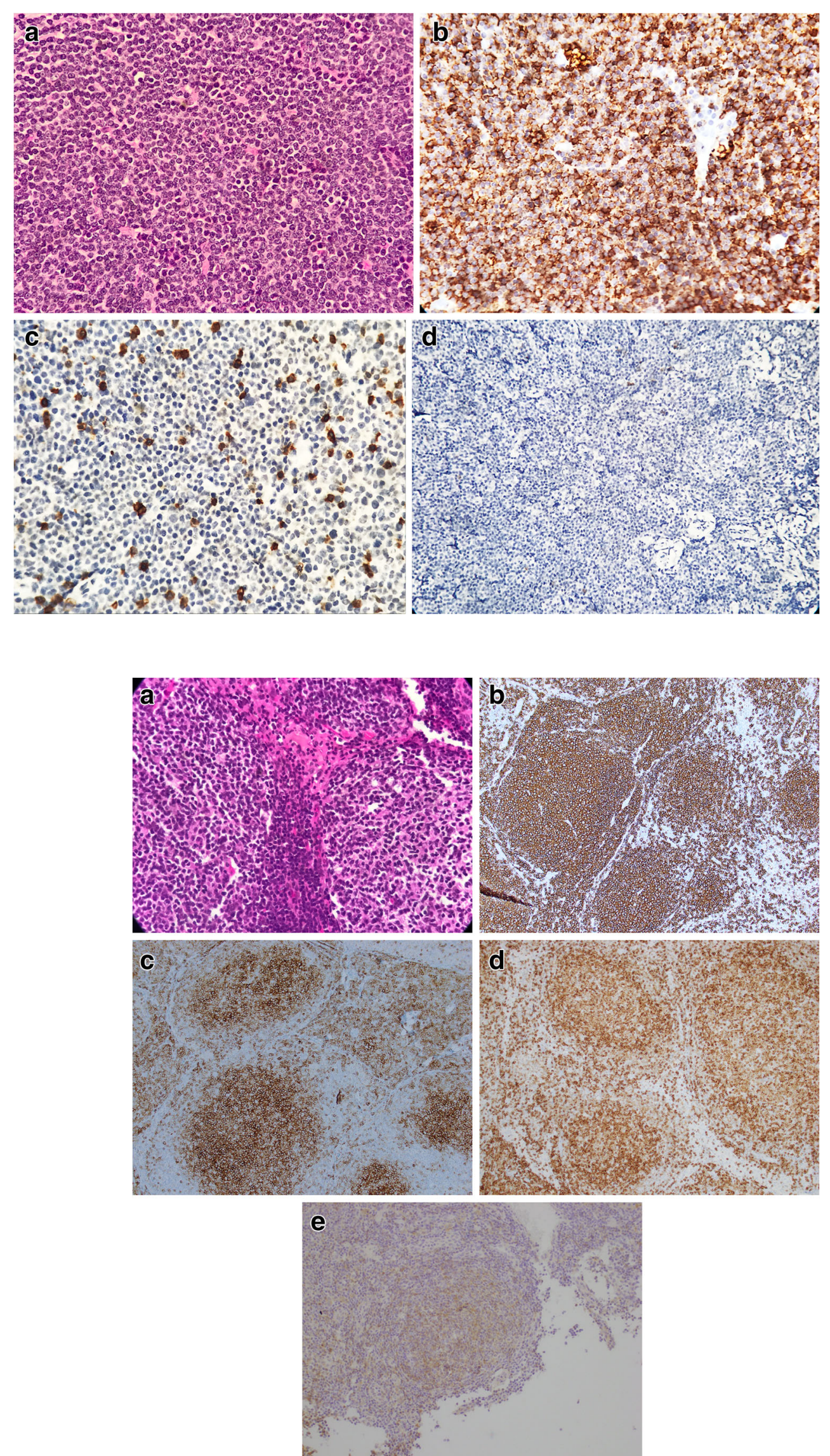
Fig. 4 Mantle cell lymphoma. a Mantle cell lymphoma involving a lymph node (hematoxylin and eosin), $\mathbf{b}$ the cells are positive for CD20, and cyclin-D1. d CD180 is negative (original magnification $\times 400$ )

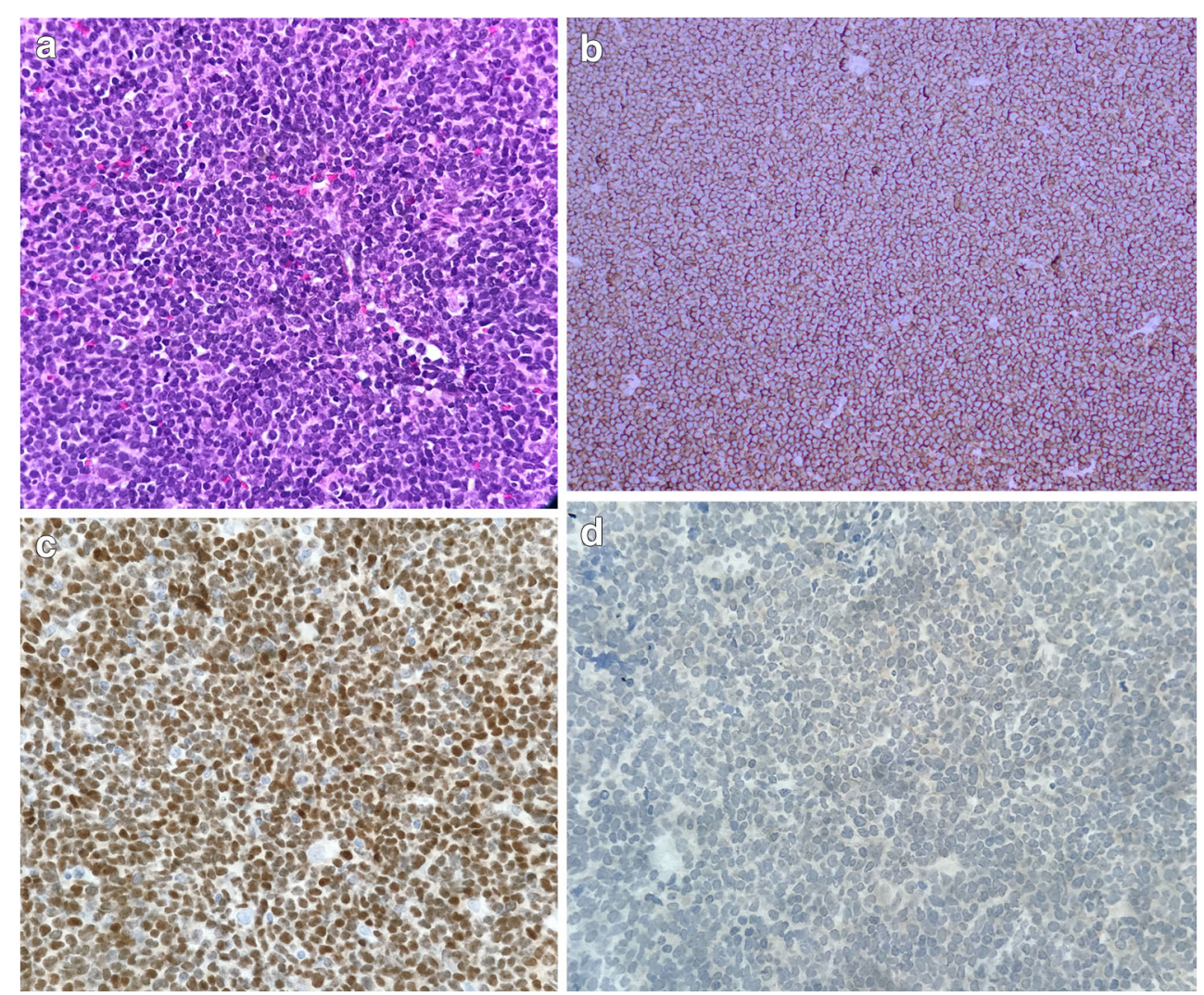

and overlap with other subtypes of low-grade cell lymphoma on the other hand, the demand for specific markers in the era of precision medicine is increasing.
Potential advantages of using immunohistochemistry over flow cytometry is the relative ease of use by general pathologists, lower cost, and the ability to perform the assay on
Fig. 5 Diffuse large B cell lymphoma (DLBCL). a DLBCL composed of large cells with fine chromatin and prominent nucleoli. b The neoplastic cells are positive for PAX5 and $\mathbf{c}$ CD10. $\mathbf{d}$ The cells are negative for CD180
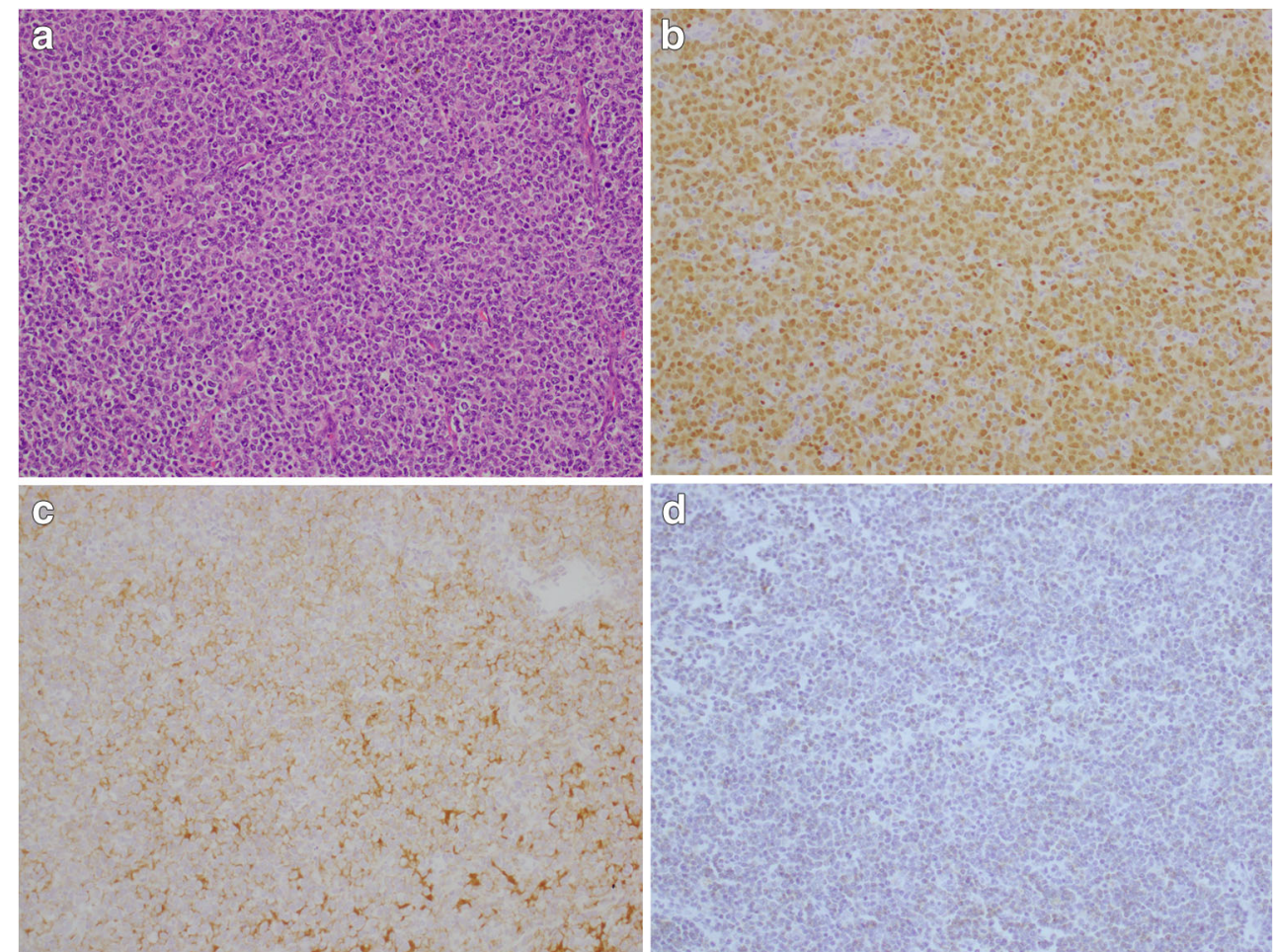
paraffin-embedded, formalin-fixed tissues, including archived material.

All the follicular lymphoma cases in our study are low grade and show predominantly follicular pattern. While CD180 is expressed in marginal zone lymphoma as well as nodal cells in follicular lymphoma, two entities that can show some morphological overlap, the resolution of this differential diagnosis can be achieved rather easily using CD10, CD21, BCL6, GCET, and LMO1, which are typically positive in FL and negative in MZL. Of note, myeloid nuclear differentiation antigen (MNDA) is positive in two thirds of MZL cases and less than $5 \%$ of FL cases [13]. The integrative approach in the diagnosis of lymphoma using clinical, radiological, morphological, immunohistochemical, and molecular studies is essential to accurately diagnose low-grade lymphoma, which in turn could affect treatment and prognosis. Studies with larger numbers of specimens and studies to elucidate CD180 expression in other types of lymphoma (such as lymphoplasmacytic lymphoma) are essential to further characterize the role of CD180 immunohistochemical stain in the routine clinical practice. Validation of the intended antibodies and clones is also essential before implementing this marker in clinical practice.

Toll-like receptors are part of the innate immune system that functions via identifying conserved motifs and cell activation by downstream signaling. While CD180 is thought of as a TLR4 member, there are important differences between CD180 and other TLR4 proteins. Firstly, the ligand-bound CD180 has a unique homodimer configuration that is different from any other TLR [14]; secondly, CD180 lacks the component necessary for downstream activation and, thus, has to associate with a mediator, specifically PIM-1L kinase. PIM1IL kinase is overexpressed and upregulated on certain tumor cells, specifically, lymphoma, leukemia, and prostate adenocarcinoma [2]. Therefore, inhibition of PIM kinase can be a potential target for therapy in these tumors, and CD180 can be used as a surrogate marker for the response of these tumors to said therapy.

One other interesting aspect of CD180 is the possibility of its use as a therapeutic target in treatment of lymphoma as well as its use as a marker for drug efficacy, specifically, BRD4 inhibitors [15]; the studies on these drugs are still in their initial phases and the exact role flow cytometry and immunohistochemistry in these regards are yet to be fully discovered.

\section{Conclusions}

We have demonstrated that examining the expression of CD180 using immunohistochemistry can be of help in accurately classifying low-grade B cell neoplasms. Among common types of B cell lymphoma, CD180 is expressed in marginal zone lymphoma and follicular lymphoma, and negative in other common types such as CLL/SLL, MCL, and DLBCL. The use of this marker on paraffin-embedded formalin-fixed tissue offers convenience, low cost, and better availability to the general pathologist, especially with small tissue samples.

Acknowledgements We would like to thank the laboratory personnel at Jordan University Hospital, especially Mr. Ahmad Al-Hisa, for their tremendous help in the preparation and performance of this study. Additionally, we would like to thank medical students at the University of Jordan-School of Medicine Shadad Iqneibi, Basma Owda, and Runa Amoudi for their help during this project. We would like to extend our gratitude for the Deanship of Scientific Research at the University of Jordan for providing funding for this research.

Funding Financial support was provided by the Deanship for Scientific Research at the University of Jordan.

Data availability Not applicable.

\section{Compliance with ethical standards}

Conflict of interest The authors declare that they have no conflict of interest.

Ethics approval The study was approved by the IRB at the University of Jordan and King Hussein Cancer Center.

Consent to participate Not applicable.

Consent for publication Not applicable.

Code availability Not applicable.

\section{References}

1. Divanovic S, Trompette A, Atabani SF, Madan R, Golenbock DT, Visintin A, Finberg RW, Tarakhovsky A, Vogel SN, Belkaid Y, Kurt-Jones EA, Karp CL (2005) Negative regulation of Toll-like receptor 4 signaling by the Toll-like receptor homolog RP105. Nat Immunol 6(6):571-578

2. Egli N, Zajonz A, Burger MT, Schweighoffer T (2015) Human CD180 transmits signals via the PIM-1L kinase. PLoS One 10(11):e0142741

3. Chaplin JW, Kasahara S, Clark EA, Ledbetter JA (2011) AntiCD180 (RP105) activates B cells to rapidly produce polyclonal Ig via a T cell and MyD88-independent pathway. J Immunol 187(8): 4199-4209

4. Chaplin JW, Chappell CP, Clark EA (2013) Targeting antigens to CD180 rapidly induces antigen-specific IgG, affinity maturation, and immunological memory. J Exp Med 210(10):2135-2146

5. Miguet L, Lennon S, Baseggio L, Traverse-Glehen A, Berger F, Perrusson N, Chenard MP, Galoisy AC, Eischen A, MayeurRousse C, Maar A, Fornecker L, Herbrecht R, Felman P, van Dorsselaer A, Carapito C, Cianférani S, Mauvieux L (2013) Cellsurface expression of the TLR homolog CD180 in circulating cells from splenic and nodal marginal zone lymphomas. Leukemia 27(8):1748-1750

6. Mayeur-Rousse C, Guy J, Miguet L, Bouyer S, Geneviève F, Robillard N, Solly F, Maar A, Bené MC, Mauvieux L, on behalf of the GEIL (Groupe d'Etude Immunologique des Leucémies) and 
GEIL (Groupe d'Etude Immunologique des Leucémies) (2016) CD 180 expression in B-cell lymphomas: a multicenter GEIL study. Cytometry B Clin Cytom 90(5):462-466

7. Wu J, Sheng Y, Sui JN, Weng XQ (2018) Characteristics of CD180 expression and its diagnostic value in B cell chronic lymphoproliferative disorders. Zhongguo shi yan xue ye xue za zhi 26(6):18111815

8. Mestrallet F, Sujobert P, Sarkozy C, Traverse-Glehen A, CalletBauchu E (2016) CD180 overexpression in follicular lymphoma is restricted to the lymph node compartment. Cytometry B Clin Cytom 90(5):433-439

9. Swerdlow SH, Campo E, Harris NL, Jaffe ES, Pileri SA et al (2017) WHO Classification of Tumours of Haematopoietic and Lymphoid Tissues. Revised 4th ed. International Agency for Research on Cancer (IARC), Lyon, pp 407-410

10. Ikeda JI, Kohara M, Tsuruta Y, Nojima S, Tahara S, Ohshima K, Kurashige M, Wada N, Morii E (2017) Immunohistochemical analysis of the novel marginal zone B-cell marker IRTA1 in malignant lymphoma. Hum Pathol 59:70-79

11. Lachar WA, Shahab I, Saad AJ (2007) Accuracy and costeffectiveness of core needle biopsy in the evaluation of suspected lymphoma: a study of 101 cases. Arch Pathol Lab Med 131(7): 1033-1039

12. Hu Q, Naushad H, Xie Q, Al-Howaidi I, Wang M, Fu K (2013) Needle-core biopsy in the pathologic diagnosis of malignant lymphoma showing high reproducibility among pathologists. Am J Clin Pathol 140(2):238-247

13. Wang Z, Cook JR (2019) IRTA1 and MNDA expression in marginal zone lymphoma: utility in differential diagnosis and implications for classification. Am J Clin Pathol 151(3):337-343

14. Yoon SI, Hong M, Wilson IA (2011) An unusual dimeric structure and assembly for TLR4 regulator RP105-MD-1. Nat Struct Mol Biol 18(9):1028-1035

15. Yeh TC, O'Connor G, Petteruti P, Dulak A, Hattersley M, Barrett JC, Chen H (2017) Identification of CCR2 and CD180 as robust pharmacodynamic tumor and blood biomarkers for clinical use with BRD4/BET inhibitors. Clin Cancer Res 23(4):1025-1035

Publisher's note Springer Nature remains neutral with regard to jurisdictional claims in published maps and institutional affiliations. 